\title{
Preface
}

\section{4th IWR Special Issue on Scientific Computing}

\author{
Hans Georg Bock ${ }^{1}$ - Vincenzo Capasso ${ }^{2}$. Willi Jäger ${ }^{1} \cdot$ Hoang Xuan Phu $^{3}$ \\ Published online: 8 March 2021 \\ (C) Vietnam Academy of Science and Technology (VAST) and Springer Nature Singapore Pte Ltd. 2021
}

Whereas the Life Sciences were fields historically concerned largely with qualitative understanding of phenomena and inferring processes from patterns, the present avalanche of data in particular at the molecular and cellular level and the progress in computer technology have encouraged a more quantitative approach beginning from information on processes at the micro and even nano level.

Bert Sakmann (Neuroscientist, Cell Physiologist; Nobel Laureate in Medicine 1991) stated already years ago: "We have plenty of data, we urgently need modelling and simulation. The scene for more quantitatively oriented biosciences has to be pre-pared." He strongly supported IWR in promoting the cooperation of Mathematical and Computational Sciences with Life Sciences.

Modern computers allow processing and analyzing huge data sets, but also facilitate numerical simulation based on mathematical models of physical, chemical, biological and medical processes. Theoretically founded modelling and simulations are providing new

Vincenzo Capasso

vincenzo.capasso@unimi.it

Hans Georg Bock

Bock@iwr.uni-heidelberg.de

Willi Jäger

jaeger@iwr.uni-heidelberg.de

Hoang Xuan Phu

hxphu@math.ac.vn

1 Interdisciplinary Center for Scientific Computing (IWR), Heidelberg University, Im Neuenheimer Feld 205, 69120 Heidelberg, Germany

2 ADAMSS (Centre for Advanced Applied Mathematical and Statistical Sciences), Universitá degli Studi di Milano La Statale, 20133 Milano, Italy

3 Institute of Mathematics, Vietnam Academy of Science and Technology, 18 Hoang Quoc Viet Road, Hanoi, Vietnam 
insights into the microscopic building blocks and modules of life and into the interactions in complex biological and biomedical systems. They also help to solve challenging problems in biotechnology, in medical diagnostics, therapy and prevention and in medical technology. It is for example possible to perform realistic "in silico" experiments, which might even allow testing possible therapies, without harming animals or exposing human beings to heavy risks.

Indeed, a more direct and systematic interaction of mathematicians with biologists and physicians surely leads to a sound advancement in Life Sciences as well as in Mathematics responding to many still open scientific challenges.

The case of the current Covid-19 pandemic has evidenced the urgent need of an integrated multidisciplinary, multiscale approach for controlling and preventing events of the same kind, all over the planet. Epidemiologists are relying on mathematical models to help policy makers get ahead of the Covid-19 pandemic. But finding proper model systems for the dynamics of the virus in the cell and in the body, for its spread in a population, for the dynamics of Covid-19 as a disease, their analysis, calibration, simulation and validation are huge challenges. The leap from equations to decisions is a long one.

This issue, the 4th IWR Special Issue on Scientific Computing, includes a series of contributions at the interface between Mathematical and Computational Sciences and Life Sciences, offering a snapshot of various fields of application of modelling, analysis and computational issues. As the pandemic started in 2020, in the year in which the selection of the present contributions took place, the topics do not relate directly to specific Covid-19 problems.

Our hope is to contribute to building bridges between Mathematics and Life Sciences. Let us finally refer the readers of this volume to the point of view of the physicist John L. Synge, according to whom:

"Nature does not offer her problems ready formulated. They must be dug out by pick and shovel, and he who will not soil his hands will never see them." [The American Mathematical Monthly, 1944, Vol. 51, No. 4, p. 186].

Publisher's Note Springer Nature remains neutral with regard to jurisdictional claims in published maps and institutional affiliations. 